\title{
ANALYTICITY OF SOLUTIONS AND KOLMOGOROV'S DISSIPATION SCALE FOR 2D NAVIER-STOKES EQUATIONS
}

\author{
ARMEN SHIRIKYAN \\ Department of Mathematics, Heriot-Watt University \\ Edinburgh EH14 4AS, Scotland, UK \\ E-mail: A.Shirikyan@ma.hw.ac.uk
}

1. Introduction. Let us consider the two-dimensional Navier-Stokes (NS) system on a torus:

$$
\dot{u}-\nu \Delta u+(u, \nabla) u-\nabla p=\eta(t, x), \quad \operatorname{div} u=0, \quad x=\left(x_{1}, x_{2}\right) \in \mathbf{T}^{2} .
$$

Here $\mathbf{T}^{2}=\mathbf{R}^{2} / 2 \pi \mathbf{Z}^{2}, \nu>0$ is the viscosity, $u=u(t, x)$ is the velocity field, $p$ is the pressure, and $\eta$ is an external force. Equation (1) is supplemented with the initial condition

$$
u(0, x)=u_{0}(x) .
$$

As is known [L], the problem (1), (2) is well-posed. Namely, for any right-hand side $\eta$ and initial function $u_{0}$ that belong to appropriate functional classes there is a unique solution $u(t, x)$ for $(1),(2)$. The aim of this article is to study analyticity of solutions regarded as functions of $x$ and to find an asymptotic lower bound for the radius analyticity as $\nu \rightarrow 0$.

This problem is closely related to the Kolmogorov-Obukhov hypothesis on the behaviour of the energy spectrum of solutions in the turbulent regime. More precisely, let us expand a solution of (1) into the Fourier series,

$$
u(t, x)=\sum_{j \in \mathbf{Z}^{2}} u_{j}(t) e^{i j x},
$$

and define the energy corresponding to a wave number $k$ by the formula

$$
E_{k}=\sum_{k-1 / 2 \leq|j| \leq k+1 / 2}\left|u_{j}\right|^{2} .
$$

The research was supported by EPSRC, grant GR/N63055/01.

Current address: Laboratoire de Mathématiques, Université de Paris-Sud XI, Bâtiment 425, 91405 Orsay Cedex, France, E-mail: Armen.Shirikyan@math.u-psud.fr.

2000 Mathematics Subject Classification: Primary 35Q30; Secondary 60H15.

The paper is in final form and no version of it will be published elsewhere. 
Roughly speaking, in the $2 \mathrm{D}$ case, the hypothesis is that there is a threshold $\lambda_{2 \mathrm{D}}=\lambda_{2 \mathrm{D}}(\nu)$ called Kolmogorov's dissipation scale such that

$$
E_{k} \sim k^{-3} \quad \text { for } k \leq \lambda_{2 \mathrm{D}}^{-1}, \quad E_{k} \lesssim k^{-N} \quad \text { for } k \geq \lambda_{2 \mathrm{D}}^{-1},
$$

where $N>0$ is an arbitrary constant. Furthermore, the Kolmogorov dissipation scale is of order $\nu^{1 / 2}$.

Let us consider a solution for (1) that admits analytic continuation to the domain $\left|\operatorname{Im} x_{i}\right| \leq r \nu^{\gamma}, i=1,2$, where $r>0$ is a constant. In this case, we have

$$
\left|u_{j}\right| \leq \text { const. } e^{-r \nu^{\gamma}|j|}
$$

whence it follows that $E_{k} \lesssim e^{-r \nu^{\gamma} k}$. Therefore,

$$
E_{k} \lesssim k^{-N} \quad \text { for } \frac{k}{\ln k} \gtrsim \nu^{-\gamma}
$$

Hence, ignoring the logarithm, one can say that, if the Kolmogorov-Obukhov hypothesis is true, then $\lambda_{2 \mathrm{D}} \gtrsim \nu^{\gamma}$. Thus, an asymptotic estimate for the radius of analyticity implies a lower bound for the Kolmogorov dissipation scale. See [Fr, Ga, HKR, Ku] and references therein for a more detailed discussion.

The problem of analyticity of solutions for deterministic Navier-Stokes equations was studied in many papers (see, e.g., [FT, HKR]). The aim of this article is to present some results in the case when the right-hand side $\eta$ is a random process analytic in the space variables and white in time. This case was investigated earlier in [M, BKL]. In particular, as is shown in [BKL], for any initial function $u_{0}$ the solution of the problem (1), (2) is analytic in $x$ for $t>0$, and its radius of analyticity $\rho_{\nu}$ can be estimated asymptotically from below by $\nu^{3+\delta}$ for any $\delta>0$. Our estimates for solutions of (1), (2) imply that $\rho_{\nu} \gtrsim \nu^{2+\delta}$ for any $\delta>0$. We note that this assertion is true for any stationary solution of Eq. (1).

2. Preliminaries. In this section, we introduce necessary functional spaces, recall the definition of a solution for Eq. (1) and the notion of a stationary solution, and formulate some known results.

Let $H^{s}=H^{s}\left(\mathbf{T}^{2}, \mathbf{R}^{2}\right)$ be the space of vector functions $u=\left(u_{1}, u_{2}\right)$ on $\mathbf{T}^{2}$ whose components belong to the Sobolev space of order $s$. For $s=0$, we obtain the usual space $L^{2}=L^{2}\left(\mathbf{T}^{2}, \mathbf{R}^{2}\right)$ with natural norm $|\cdot|$. Let $H$ be the subspace of $u \in L^{2}$ such that $\operatorname{div} u=0$ and $\int_{\mathbf{T}^{2}} u(x) d x=0$ and let $\Pi: L^{2} \rightarrow H$ be the orthogonal projection onto $H$.

Applying $\Pi$ to Eq. (1), we write it in the form (see $[\mathrm{VF}]$ )

$$
\dot{u}+\nu L u+B(u, u)=\eta(t),
$$

where $L$ is the restriction of the operator $-\Delta$ to $H, B(u, u)=\Pi(u, \nabla) u$ is the nonlinear term, and $\eta(t)$ is the projection of the external force. (To simplify the notation, we denote the external force and its projection to $H$ by the same symbol.)

To describe the class of right-hand sides for (3), we introduce a trigonometric basis in $H$. Namely, let $\mathbf{Z}^{\prime}$ be a subset of $\mathbf{Z}_{0}^{2}=\mathbf{Z}^{2} \backslash\{0\}$ such that $\mathbf{Z}^{\prime} \cup\left(-\mathbf{Z}^{\prime}\right)=\mathbf{Z}_{0}^{2}$ and let

$$
e_{j}(x)=\frac{\sin (j x) j^{\perp}}{\sqrt{2} \pi|j|}, \quad e_{-j}(x)=\frac{\cos (j x) j^{\perp}}{\sqrt{2} \pi|j|}, \quad j=\left(j_{1}, j_{2}\right) \in \mathbf{Z}^{\prime},
$$


where $j^{\perp}=\left(-j_{2}, j_{1}\right)$ and $|j|=\left(j_{1}^{2}+j_{2}^{2}\right)^{1 / 2}$. It is clear that $L e_{j}=|j|^{2} e_{j}, j \in \mathbf{Z}_{0}^{2}$, and that $\left\{e_{j}, j \in \mathbf{Z}_{0}^{2}\right\}$ is a basis in $H$.

We assume that $\eta$ has the form

$$
\eta(t, x)=\frac{\partial}{\partial t} \zeta(t, x), \quad \zeta(t, x)=\sum_{j \in \mathbf{Z}_{0}^{2}} b_{j} \beta_{j}(t) e_{j}(x),
$$

where $\beta_{j}$ are independent standard Brownian motions defined on a complete probability $(\Omega, \mathcal{F}, \mathbf{P})$ with filtration $\mathcal{F}_{t}$ and $b_{j}$ are real constants satisfying the condition

$$
\sum_{j \in \mathbf{Z}_{0}^{2}}|j|^{2} b_{j}^{2}<\infty
$$

This assumption implies, in particular, that almost all sample paths $\zeta(t, \cdot)$ belong to the space $C\left(\mathbf{R}_{+}, V\right)$ of continuous functions on the half-line $\mathbf{R}_{+}=[0,+\infty)$ with range in $V:=H \cap H^{1}\left(\mathbf{T}^{2}, \mathbf{R}^{2}\right)$. Equation (3) is regarded as an Itô's stochastic PDE.

Let $L_{\text {loc }}^{2}\left(\mathbf{R}_{+}, V\right)$ be the space of Bochner-measurable functions $u(t): \mathbf{R}_{+} \rightarrow V$ such that $\int_{0}^{T}\|f(t)\|^{2} d t<\infty$ for any $T>0$, where $\|u\|=\left|L^{1 / 2} u\right|$ is the norm in $V$.

Definition 1. A random process $u(t)=u(t, x)$ in $H$ defined on the half-line $t \geq 0$ and progressively measurable with respect to $\mathcal{F}_{t}$ is called a strong solution of Eq. (3) if the following two conditions hold with probability 1.

(i) The function $u(t, x)$ belongs to $L_{\text {loc }}^{2}\left(\mathbf{R}_{+}, V\right) \cap C\left(\mathbf{R}_{+}, H\right)$.

(ii) For any $t>0$,

$$
u(t)+\int_{0}^{t}(\nu L u+B(u, u)) d s=u(0)+\zeta(t),
$$

where the left- and right-hand sides of this relation are regarded as elements of the space $H^{-1}\left(\mathbf{T}^{2}, \mathbf{R}^{2}\right)$.

A proof of the following result can be found in [VF, Chapter X] (also see [DaZ, Chapter 15]).

Proposition 2. Suppose that condition (5) holds. Then for any $\mathcal{F}_{0}$-measurable random variable $u_{0}$ with range in $H E q$. (3) has a unique solution on $[0, \infty)$ that satisfies the initial condition (2).

Let $u(t)$ be a solution for Eq. (3) and let $\mu(t)$ be its distribution at time $t$. Thus, $\mu(t)$ is a probability Borel measure in the functional space $H$.

Definition 3. The solution $u(t)$ is said to be stationary if $\mu(t)$ does not depend on $t$. In this case, $\mu(t) \equiv \mu$ is called a stationary measure for Eq. (3).

A proof of the following theorem can be found in [DaZ, Chapter 15].

Proposition 4. Suppose that condition (5) holds. Then Eq. (3) has a stationary measure. 
3. Main results. We begin with an estimate for a second exponential moment for stationary solutions.

Theorem 5. Suppose that condition (5) holds. There are positive constants $\sigma$ and $C$ not depending on $\nu$ such that, if $\mu$ is a stationary measure for Eq. (3) with some $\nu \in(0,1]$, then

$$
\int_{H} \exp \left(\sigma \nu\|u\|^{2}\right) \mu(d u) \leq C .
$$

We now assume that the coefficients $b_{j}$ entering the right-hand side of (3) (see (4)) satisfy the following inequality for some $\rho>0$ :

$$
\sum_{j \in \mathbf{Z}_{0}^{2}} e^{2 \rho|j|} b_{j}^{2}<\infty .
$$

Recall that, for a function $u(t, x)$ with range in $H$, we denote by $u_{j}(t)$ its Fourier coefficients. The following theorem establishes the analyticity of solutions for the Cauchy problem (3), (2) and gives an asymptotic lower bound for the radius of analyticity.

THEOREM 6. Suppose that condition (6) holds. Let $u_{0}=u_{0}^{\nu}(x)$ be a family of random initial functions that satisfy the inequality

$$
\mathbf{E} \exp \left(\sigma \nu\left\|u_{0}^{\nu}\right\|^{2}\right) \leq R \quad \text { for } 0<\nu \leq 1
$$

where the positive constants $\sigma$ and $R$ do not depend on $\nu$. Then for any $t_{0} \geq 1, T>0$ and $\delta \in(0,1]$ there are positive random variables $r_{\nu}=r_{\nu}\left(t_{0}, T, \delta\right)$ and $C_{\nu}=C_{\nu}\left(t_{0}, T, \delta\right)$ such that, with probability 1 ,

$$
\left|u_{j}(t)\right| \leq C_{\nu} e^{-r_{\nu} \nu^{2+\delta}|j|}, \quad t_{0} \leq t \leq t_{0}+T, \quad j \in \mathbf{Z}_{0}^{2} .
$$

Moreover, for any integer $m \geq 1$ there is a constant $K_{m}=K_{m}(\sigma, T, \delta, R)>0$ not depending on $t_{0}$ and $\nu$ such that

$$
\mathbf{E} r_{\nu}^{-m} \leq K_{m}, \quad \mathbf{E} C_{\nu}^{m} \leq K_{m} \nu^{-m / 2} \quad \text { for } 0<\nu \leq 1 .
$$

In particular, any solution of Eq. (3) with deterministic initial function $u_{0} \in V$ is analytic in $x$ with probability 1 , and its radius of analyticity can be estimated from below by $r_{\nu} \nu^{2+\delta}$. Theorem 5 implies that the above assertions are valid for any stationary solution of Eq. (3).

Proofs of Theorems 5 and 6 are given in $[\mathrm{S}]$.

Acknowledgements. A part of this article was written during the Workshop on Evolution Equations at the Banach Center, Warsaw (1-6 July 2001), and I would like to thank the organisers for hospitality. I am also grateful to Prof. Sergei Kuksin for encouragement and stimulating discussions of the results of this paper.

\section{References}

[BKL] J. Bricmont, A. Kupiainen, and R. Lefevere, Probabilistic estimates for the twodimensional stochastic Navier-Stokes equations, J. Statist. Phys. 100 (2000), 743-756. 
[DaZ] G. Da Prato and J. ZabczyK, Ergodicity for Infinite-Dimensional Systems, London Math. Soc. Lecture Note Ser. 229, Cambridge University Press, Cambridge, 1996.

[FT] C. Foias and R. Temam, Gevrey class regularity for the solutions of the Navier-Stokes equations, J. Funct. Anal. 87 (1989), 359-369.

[Fr] U. Frisch, Turbulence. The Legacy of A. N. Kolmogorov, Cambridge University Press, Cambridge, 1995.

[Ga] G. Gallavotti, Foundations of Fluid Dynamics, Springer, Berlin, 2001.

[HKR] W. D. Henshaw, H.-O. Kreiss, and L. G. Reyna, Smallest scale estimates for the Navier-Stokes equations for incompressible fluids, Arch. Rational Mech. Anal. 112 (1990), $21-44$.

[Ku] S. B. KuKsIn, Spectral properties of solutions for nonlinear PDEs in the turbulent regime, Geom. Funct. Anal. 9 (1999), 141-184.

[L] J.-L. Lions, Quelques méthodes de résolution des problèmes aux limites non linéaires, Gauthier-Villars, Paris, 1969.

[M] J. C. Mattingly, The Stochastic Navier-Stokes Equation: Energy Estimates and Phase Space Contraction, Ph. D. thesis, Princeton University, 1998.

[S] A. Shirikyan, Analyticity of solutions for randomly forced two-dimensional NavierStokes equations, Uspekhi Mat. Nauk 57 (2002), no. 4, 151-166 (in Russian); English transl.: Russian Math. Surveys 57 (2002), 785-799.

[VF] M. I. VishiK and A. V. Fursikov, Mathematical Problems of Statistical Hydromechanics, Kluwer, Dordrecht, 1988. 